\title{
The Effect of Discussions About Advance Directives on Patients' Satisfaction with Primary Care
}

\author{
William M. Tierney, MD, Paul R. Dexter, MD, Gregory P. Grame/spacher, MD,
} Anthony J. Perkins, MS, Xiao-Hua Zhou, PhD, Fredric D. Wolinsky, PhD

BACKGROUND: Discussions of end-of-life care should be held prior to acute, disabling events. Many barriers to having such discussions during primary care exist. These barriers include time constraints, communication difficulties, and perhaps physicians' anxiety that patients might react negatively to such discussions.

OBJECTIVE: To assess the impact of discussions of advance directives on patients' satisfaction with their primary care physicians and outpatient visits.

DESIGN: Prospective cohort study of patients enrolled in a randomized, controlled trial of the use of computers to remind primary care physicians to discuss advance directives with their elderly, chronically ill patients.

SETTING: Academic primary care general internal medicine practice affiliated with an urban teaching hospital.

PARTICIPANTS: Six hundred eighty-six patients who were at least 75 years old, or at least 50 years old with serious underlying disease, and their 87 primary care physicians (57 residents, 30 faculty general internists) participated in the study.

MEASUREMENTS AND MAIN RESULTS: We assessed patients' satisfaction with their primary care physicians and visits via interviews held in the waiting room after completed visits. Controlling for satisfaction at enrollment and physician, patient, and visit factors, discussing advance directives was associated with greater satisfaction with the physician $(P=.052)$. At follow-up, the strongest predictor of satisfaction with the primary care visit was having previously discussed advance directives with that physician $(P=.004)$, with a trend toward greater visit satisfaction when discussions were held during that visit $(P=.069)$. The percentage of patients scoring a visit as "excellent" increased from $34 \%$ for visits prior without advance directive discussions to $51 \%$ for visits with such discussions $(P=.003)$.

CONCLUSIONS: Elderly patients with chronic illnesses were more satisfied with their primary care physicians and outpatient visits when advanced directives were discussed. The improvement in visit satisfaction was substantial and persistent. This should encourage physicians to initiate such discussions to overcome communication barriers that might

Received from the Department of Medicine, Indiana University School of Medicine (WMT, PRD, GPG, X-HZ); Wishard Memorial Hospital (WMT, PRD, GPG); the Regenstrief Institute for Health Care (WMT, PRD, AJP, X-HZ); the Roudebush VA Medical Center (WMT, PRD) Indianapolis, Ind; and the School of Public Health, Saint Louis University, St. Louis, Mo (FDW).

Address correspondence and reprint requests to Dr. Tierney: Roudebush VA Medical Center (11H), 1481 West Tenth St., Indianapolis, IN 46202 (e-mail: wtierney@iupui.edu). result in reduced patient satisfaction levels.

KEY WORDS: patient satisfaction; advance directives; endof-life care; primary care. J GEN INTERN MED 2001;16:32-40.

A dvance directives can help patients control health care decisions in cases where they may be unable to speak for themselves. Although they are being advocated by supporters of patient autonomy, ${ }^{1-3}$ advance directives have also been endorsed as a means for controlling the high costs of health care at the end of life. ${ }^{4,5}$

Both patients and physicians feel that advance directives are important and should be discussed., ${ }^{1,6}$ Yet we, ${ }^{9}$ as well as others, ${ }^{1,7,8}$ have shown that such discussions are uncommon. Patients often feel it is their physicians' responsibility to initiate advance care discussions, while physicians believe the subject should be raised by their patients. ${ }^{1,6}$ Additional barriers include time constraints, language and other impediments to communication, and perhaps a concern by the physician that patients would be troubled by these discussions. Prior research has suggested that completing advance directives may not be associated with the subsequent care provided (and its costs) or patients' satisfaction with their care. ${ }^{10-13}$ Will patients worry that they are sicker than their physicians have led them to believe? Or in managed care environments, will there be suspicion that the physician has a financial conflict of interest and is trying to decrease costs by limiting care? With increasing emphasis on patient satisfaction and clinical productivity, physicians may decide not to initiate time-consuming advance care discussions, especially if such discussions might upset their patients.

In a randomized, controlled trial published previously, ${ }^{9}$ we demonstrated that computer-generated reminders increased discussions of end-of-life care between academic physicians and their primary care patients and the completion of advance directive forms. During that study, we prospectively assessed patients' satisfaction with their primary care physicians and scheduled outpatient visits.

\section{METHODS}

\section{Study Site}

This study was approved by the Indiana University Institutional Review Board. The data for this report 
came from a randomized, controlled trial of computergenerated reminders, displayed to primary care physicians, to discuss advance directives with their scheduled patients. ${ }^{9}$ Briefly, the trial was conducted in a hospital-based academic primary care general internal medicine practice that mainly serves inner-city indigent patients. It is staffed by general internal medicine faculty and residents of the Indiana University School of Medicine. Residents attend their primary care practices one half-day per week while faculty attend from one to four half-days a week. Each physician maintains a panel of patients for whom he or she provides primary care (roughly 100 to 200 patients per physician per weekly half-day session attended). For their entire residencies, residents assume primary care decision making for their assigned patients, although they must briefly present each patient to a faculty internist (also practicing at the same site) after each visit. Approximately half of the patients have faculty internists as their primary care physicians.

At the time of this study, adult primary care was provided in four parallel practices, each of which met for eight half-day sessions per week. Since 1981, new residents have been randomly assigned to open practice sessions. ${ }^{14}$ The computer system used in this study has generated reminders to increase physician compliance with primary care guidelines for more than 25 years. ${ }^{15,16}$ For this study, the 32 half-day sessions were randomly assigned to one of four groups: (1) computer reminders to discuss instruction directives (a list of care interventions which the patient wanted or did not want in the case of terminal illness and cognitive impairment); (2) reminders to discuss proxy directives (a form for naming a health care representative); (3) reminders to discuss both types of advance directives; and (4) control (i.e., no advance directive reminders).

\section{Subject Enrollment and Data Collection}

To obtain an enriched sample of patients for whom advance directives are currently relevant, we attempted to enroll all scheduled patients who had been active within this practice for at least a year and were either at least 75 years old or between 50 and 74 years old with one of the following chronic, morbid conditions: ischemic heart disease, chronic heart failure, chronic obstructive pulmonary disease, cerebrovascular disease, cancer (other than nonmelanomatous skin cancer), chronic renal insufficiency, and chronic liver failure. Eligible patients, identified using information stored in their electronic medical records, were approached by research assistants in the waiting room either before or after a scheduled visit with their primary care physicians. Those able to converse in English were first asked if they had previously completed an advance directive form with their current primary care physician; if so, they were excluded from the study. This was done because the primary dependent variable of the controlled trial of computer reminders was completion of advance directive forms. The remaining eligible patients who were willing to participate were administered the Pfeiffer Short Portable Mental Status Questionnaire. ${ }^{17}$ Those passing this cognition screen (using standard scoring criteria) and agreeing to participate underwent a baseline evaluation that included two measures of patient satisfaction: (1) the 10-item instrument developed by the American Board of Internal Medicine (ABIM), ${ }^{18}$ which assesses patient satisfaction with the primary care physician, and (2) the Visit-Specific Questionnaire developed for the Medical Outcomes Study (MOS-VSQ) $^{19}$ that assesses patients' satisfaction with a particular visit. Each item of both scales has a standard 5 -point Likert response from 1 (excellent) to 5 (poor) (see Appendix). For this study, we limited the 7-item MOS-VSQ a priori to five questions, deleting two items dealing with the practice site or the time required to schedule an appointment because neither of these was relevant to our controlled trial's hypothesis. Both the ABIM and MOS instruments had been intensively studied in this practice where factor analysis yielded, as expected, a single highly reliable scale for each instrument. ${ }^{20}$ By using both instruments, we were able to study satisfaction with the traits of the physician (predominantly communication skills) and satisfaction with characteristics the individual primary care visit.

To control for patients' health status, we administered the SF-36 at enrollment. ${ }^{21}$ For this analysis, we used the physical component score and the mental component score as summary measures. Because we expected that patients' baseline desires for end-of-life care would affect their opinions of discussions about advance directives, we assessed patients' desires for end-of-life care with an instrument created by Danis et al. ${ }^{22}$ This instrument asks which of 6 specific treatments (e.g., resuscitation, mechanical ventilation, artificial nutrition) the patient would want if he or she were terminally ill and unable to participate in clinical decision making. Each item is given a score of 1 if wanted, -1 if not wanted, and 0 if unsure. The scores are added, with an overall score ranging from -6 (least aggressive) to +6 (most aggressive).

We also assessed patients' educational attainment, which has also been shown to correlate with satisfaction, recording the patient's highest school grade completed. We also postulated that the primary care physician's age, gender, educational status (resident vs faculty), and country of medical education might correlate with both discussions of advance directives and patient satisfaction. To control for familiarity between the patients and their primary care physicians, we also extracted from patients' electronic records the number of prior outpatient encounters between each enrolled patient and his or her current primary care physician. Finally, because physician time constraints may affect both patient satisfaction and the likelihood of holding advance care discussions, we also controlled our analyses for the number of patients seen by 
the physician during each half-day practice session in which the study visit occurred.

For 1 year following enrollment, research assistants attempted to interview enrolled patients in the waiting room after all scheduled visits to their primary care physicians. During these interviews, the ABIM (physician) and MOS (visit) satisfaction questionnaires were administered, after which the patients were asked whether they had discussed advance directives or any other aspect of end-of-life care with their physician that day. Responding "yes" to either question was considered as having had a discussion about advance directives.

\section{Data Analysis}

The dependent variables in our analyses were ABIM and MOS-VSQ patient satisfaction scores, which we transformed to 1 to 100 scales by subtracting the lowest possible score from the actual score and dividing the result by the maximum attainable score. For both scales, higher scale scores are associated with lower satisfaction. For ease of interpretation, we inverted both scales (i.e., subtracted transformed scores from 100). We assessed each instrument's internal consistency (reliability) using Cronbach coefficient $\alpha$.

The unit of analysis for this study was the individual scheduled visit. To control for correlations among visits by individual patients, and correlations among patients treated by the same physician, we assessed multivariable correlates of patient satisfaction by employing a hierarchical (multilevel) linear model with the physician at the top level, patient nested within physician as the second level, and visits nested within patient as the lowest level. ${ }^{23}$ These models were fitted using Proc Mixed in SAS (Version 6.12, SAS Institute, Cary, NC). We conducted separate multivariable analyses for ABIM and MOS-VSQ satisfaction scores and controlled for baseline differences between patients and physicians. These models included the patient and physician as random effects; fixed effects included variables describing the physician (gender, resident/faculty status, whether foreign educated, and intervention status in the controlled trial of computer reminders), the patient (age, gender, race, years of education, presence of any of the eligible clinical conditions, number of prior visits to their current primary care physician, Danis score, ${ }^{22}$ the SF-36 physical component score and the mental component score, and satisfaction score at baseline), and the visit. For both analyses, we included indicator variables that were scored as 1 if advance directives were discussed the day of the visit; otherwise, it was scored as 0 . To assess any persisting effects of advance directive discussions, we included another indicator variable scored as 1 if the patient had discussed advance directives with their current primary care physician at any previous visit. To roughly control for the amount of time available for such discussions, we included the number of patients seen by that physician that day. Because of limited dispersion, Danis scores were collapsed into three categories: -6 to -2 (reference), -1 to 1 , and 2 to 6 .

Patients who did not keep any scheduled visits during their 1-year study observation period were excluded from analysis. Because we wanted to control analyses for patient satisfaction at baseline (enrollment), we also excluded patients who did keep postenrollment scheduled visits but whose primary care physician at the time of enrollment left the practice (usually at the start of an academic year) prior to the patient's keeping any scheduled visits. If a patient kept at least one scheduled visit to his or her baseline physician (and patient satisfaction was assessed) but the baseline physician then left the practice, we kept in the analysis data for all visits to the baseline physician. Likewise, the small number of visits where the research assistants failed to interview the patient postvisit were also excluded. Because patients occasionally did not answer one of the questions in either of the satisfaction assessment instruments (never more than 5\% per question), we examined the effects of missing values in four ways: (1) excluding patients with any missing satisfaction data, (2) replacing the missing value with the patient sample median for that item, (3) replacing it with the mean of all items for that scale for that patient on that visit, and (4) performing multiple imputation, where missing values were modeled using other variables among cases with no missing values. Because the results were the same regardless of how we dealt with missing data, we report results where we substituted the patient's mean for all completed items for that scale.

We examined our data for evidence of multicolinearity. Only two variables among those included in the multivariable models had correlation coefficients greater than .3. Performing an ordinary least squares regression ignoring the multilevel structure of the data resulted in a maximum variance inflation factor of 1.8, indicating no important problems with colinearity. Moreover, because satisfaction data tend to be positively skewed toward patients being satisfied with their physicians, we assessed (using graphical means) the residuals at each level and found them to be normally distributed, suggesting that our results were not affected.

Finally, to provide a benchmark for visit satisfaction, we compared our results on the MOS-VSQ to those obtained by Rubin et al. ${ }^{19}$ by calculating the percentage of patients who provided an overall rating of their visit as "excellent." We then used the same modeling techniques as described above for this dependent variable.

\section{RESULTS}

During a 9-month enrollment period, 1,199 eligible patients kept primary care appointments; 1,051 (88\%) were enrolled in the study. Nine patients were excluded from analysis because they had previously completed advance 


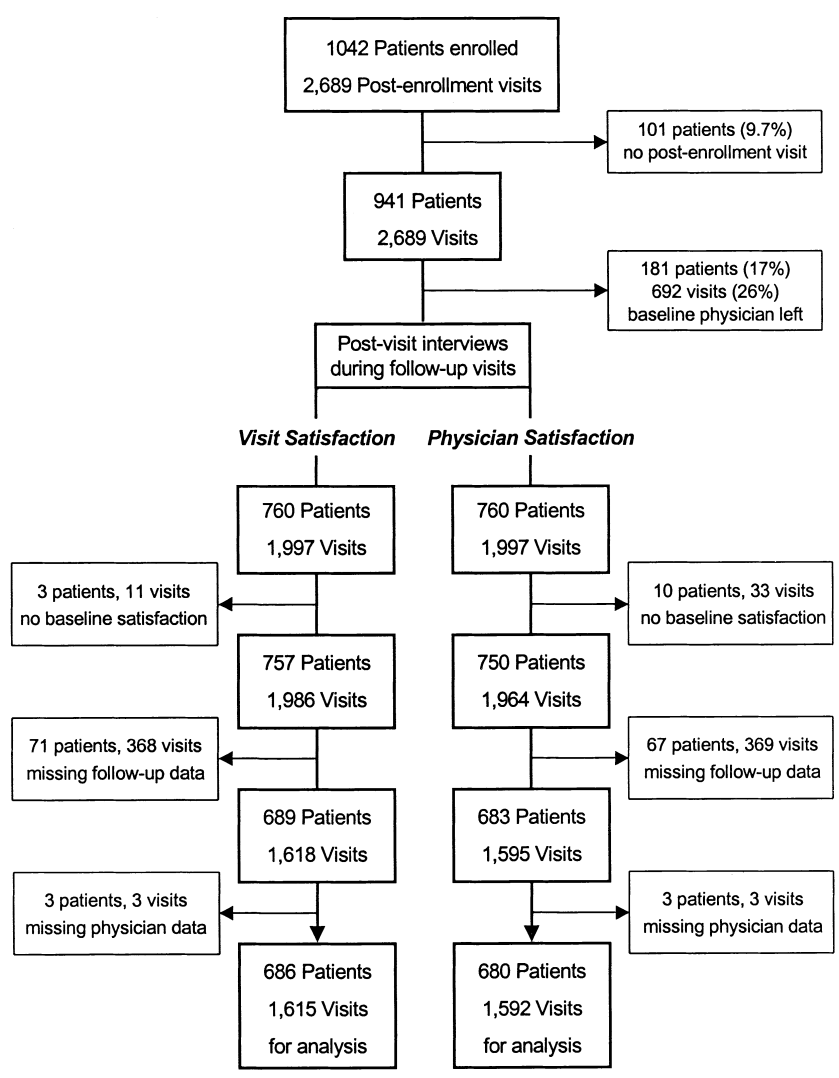

FIGURE 1. Accounting of patients and visits among patients enrolled in the original controlled trial ${ }^{9}$ and those used in this analysis.

directives with their current primary care physicians, resulting in 1,042 study patients.

As shown in Figure 1, we excluded 101 patients (9.7\%) who kept no scheduled visits to their primary care physicians during their 1-year duration in the study. Because we wished to control patient satisfaction during follow-up visits for patients' baseline satisfaction with their primary care physicians, we also excluded 181 patients (17\%) whose primary care physicians at enrollment (usually senior residents) left the practice after patient enrollment. Although such patients had 692 subsequent study visits during which satisfaction was assessed, we could not control our analyses of these visits for baseline satisfaction because all subsequent visits were made to a physician who was different from the physician for whom we assessed patient and visit satisfaction at the time of patient enrollment.

Of the remaining 760 patients who made 1,997 postenrollment scheduled visits, we excluded a small number of patients and visits $(<1 \%)$ from the analysis of satisfaction with the visit. Those excluded were missing data for satisfaction with the baseline visit or information about their physician. We also excluded $18 \%$ of the visits where the study interviewers missed the patient in the waiting room after completing these physician visits. This resulted in our excluding $9 \%$ of the remaining patients who were missed following all postenrollment visits. This yielded a final analysis set of 686 patients $(90 \%$ of those keeping scheduled study visits whose baseline physician remained in the practice) who had satisfaction assessed at 1,615 postenrollment scheduled visits. Comparing the 356 patients excluded with those not excluded from analysis (for any of the above reasons) for the variables listed in Table 1, excluded patients had significantly fewer prior visits to their baseline physician ( 1.2 vs $8.3, P<.0001)$, less heart failure $(30 \%$ vs $37 \%, P=.014)$, lower baseline physician satisfaction (77 vs $80, P=.001$ ), and lower visit satisfaction ( 73 vs $77, P=.001)$.

The 686 patients in the final analysis were cared for by 87 physicians, $33 \%$ of whom were faculty, $67 \%$ of whom were men, and $81 \%$ who graduated from U.S. medical schools. Table 1 shows descriptive data for all 686 patients, the majority of whom were women. Slightly more than half were African American, and their mean age was 65 years. Only $2 \%$ of these patients had ever discussed advance directives with their current primary care physician prior to the study. During the study, 110 (16\%) of these patients discussed advance directives or other aspects of advance care with their primary care physicians.

\section{Table 1. Patient Characteristics $(n=686)$}

\begin{tabular}{lc}
\hline Characteristic & \\
\hline Mean age \pm SD, y & $65 \pm 9.9$ \\
Female, \% & 67 \\
African American, \% & 55 \\
Education, mean years completed \pm SD & $9.4 \pm 2.8$ \\
Clinical eligibility criteria, \%* & \\
Age $\geq 75$ years & 18 \\
Ischemic heart disease & 47 \\
Chronic lung disease & 44 \\
Heart failure & 38 \\
Cerebrovascular disease & 18 \\
Cancer & 13 \\
Chronic renal insufficiency & 8 \\
Chronic liver disease & 4 \\
Danis score, \% ${ }^{\dagger}$ & \\
-6 to -2 & 43 \\
-1 to +1 & 23 \\
+2 to +6 & 34
\end{tabular}

No. of prior visits with current primary care physician (mean $\pm \mathrm{SD}$ ) $\quad 8.3 \pm 9.0$

Previously discussed advance directives with current primary care physician, \%

Baseline satisfaction with physician during the enrollment visit ${ }^{\ddagger}$

* Eligibility criteria for the original controlled trial, ${ }^{9}$ which were not mutually exclusive.

${ }^{\dagger}$ Desirability for care if terminally ill and cognitively impaired, assessed at the enrollment interview scored from -6 (least aggressive) to 0 (neutral) to +6 (most aggressive). ${ }^{17}$

${ }^{\ddagger}$ As assessed using the American Board of Internal Medicine’s questionnaire, ${ }^{14}$ transformed to a $0 \%$ to $100 \%$ scale and inverted (higher scores denote greater satisfaction).

$\S$ As assessed using the Medical Outcomes Study's visit-specific questionnaire, ${ }^{15}$ transformed to a $0 \%$ to $100 \%$ scale and inverted (higher scores denote greater satisfaction). 
The 10-item ABIM questionnaire had high internal consistency (Cronbach $\alpha=0.95$ ). Satisfaction with the physician during 1,592 postenrollment visits was, on average, high: the mean ABIM physician satisfaction score during postenrollment scheduled visits was $79 \pm$ 19 on the 100-point scale. The strongest multivariable correlate with satisfaction with the primary care physician during scheduled visits after enrollment was satisfaction with the physician during the enrollment visit, followed by the physician's seeing fewer patients that day, having some education beyond grammar school, the mental component score of the SF-36, and the physician's graduating from a U.S. medical school (Table 2). After controlling for the above factors, having discussed advance directives that day was also posi- tively correlated with satisfaction with the physician $(P=$ .052).

The 5-item MOS visit-specific questionnaire was also highly internally consistent (Cronbach $\alpha=0.90$ ). Visit satisfaction was also generally high: the mean MOS-VSQ visit satisfaction score during 1,615 postenrollment visits was $77 \pm 19$. The strongest multivariable correlate with visit satisfaction was satisfaction with the enrollment visit, followed by having previously discussed advance directives, the mental component score of the SF-36, being in the physician study group that received proxy reminders, being a high school graduate, and seeing fewer patients on the day of the visit. Discussing advance directives at that visit had borderline significance $(P=.075)$ when controlling for the above factors.

Table 2. Multivariable Correlates with Physician Satisfaction*

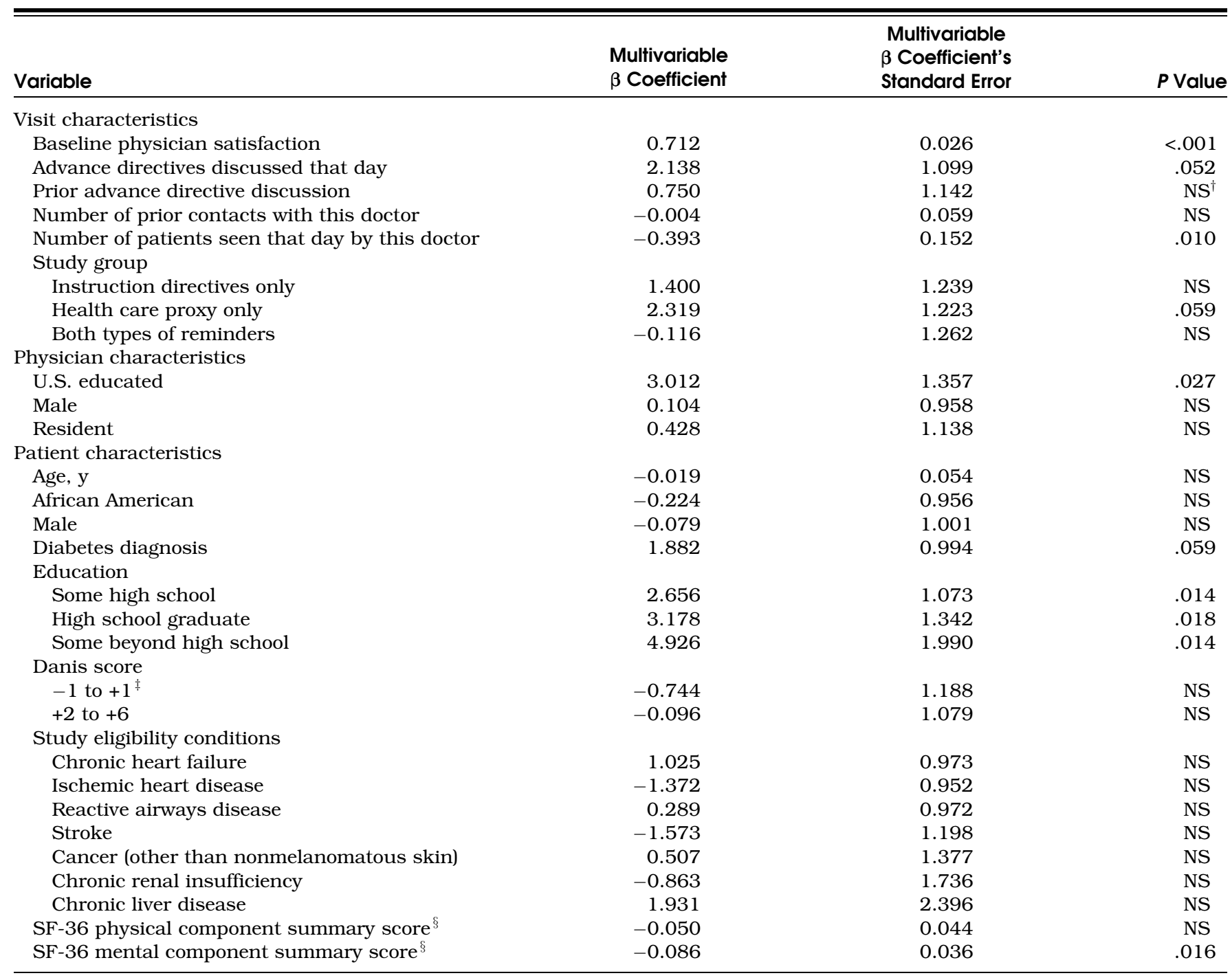

* Assessed using the American Board of Internal Medicine's survey instrument, transformed to a 0\% to 100\% scale and inverted (higher score denotes higher satisfaction). Parameter estimates were provided by hierarchical generalized linear modeling. ${ }^{18}$

${ }^{\dagger} N \mathrm{~S}$ indicates not significant $(\mathrm{P} \geq .10)$.

${ }^{\ddagger}$ Desirability for care if terminally ill and cognitively impaired, assessed at the enrollment interview (from Danis, ${ }^{17}$ with a score of -6 to - 2 as the reference category).

${ }^{\S}$ Calculated as per Ware et al. ${ }^{21}$ 
Table 3. Multivariable Correlates with Visit Satisfaction*

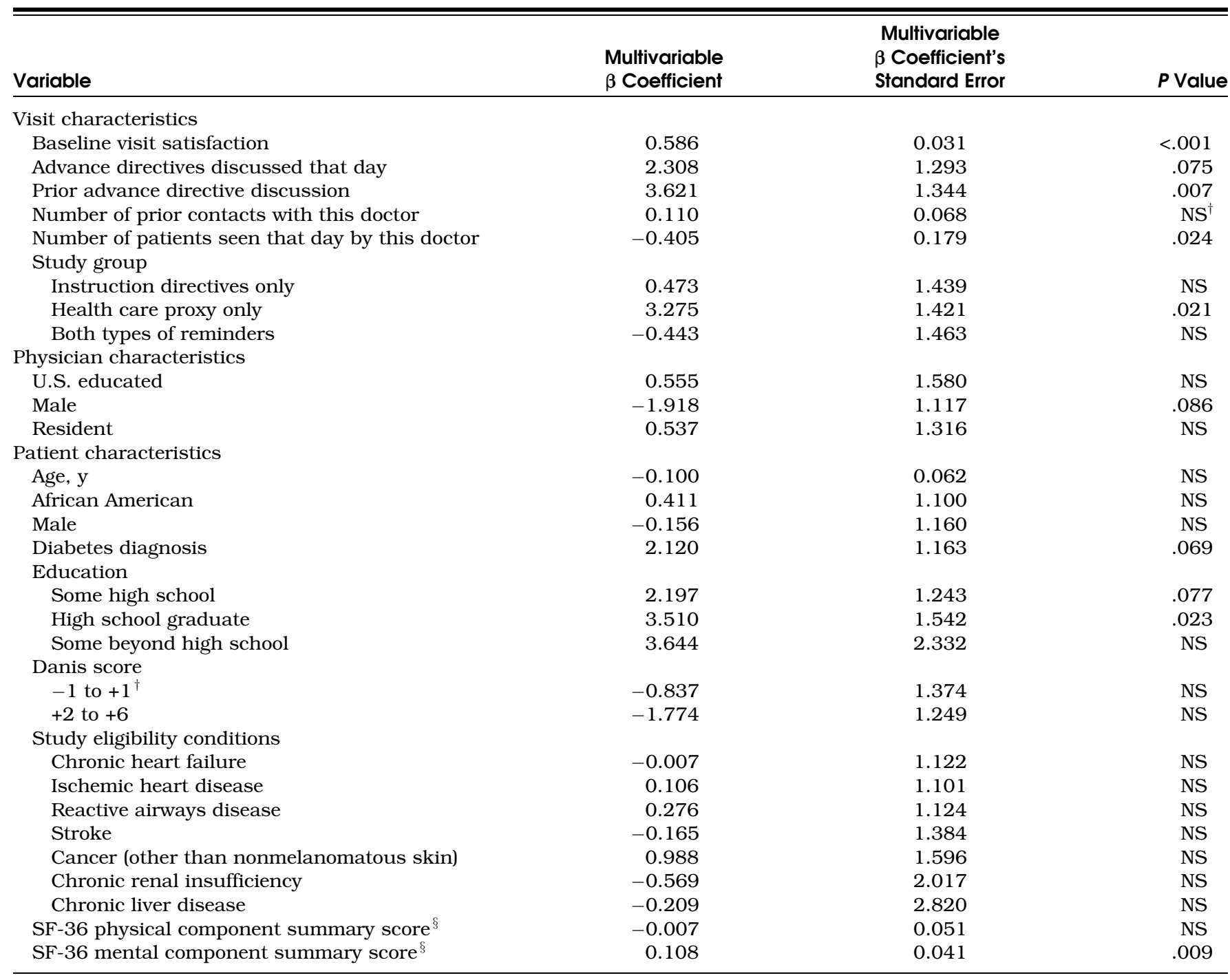

* Assessed using the Medical Outcome Study's visit-specific questionnaire, ${ }^{15}$ transformed to a 0\% to $100 \%$ scale and inverted (higher score denotes higher satisfaction). Parameter estimates were provided by hierarchical generalized linear modeling. ${ }^{18}$

${ }^{\dagger} N$ S indicates not significant $(\mathrm{P} \geq .10)$.

${ }^{\ddagger}$ Desirability for care if terminally ill and cognitively impaired, assessed at the enrollment interview (from Danis, ${ }^{17}$ with a score of -6 to - 2 as the reference category).

${ }^{\S}$ Calculated as per Ware et al. ${ }^{21}$.

We also analyzed visit satisfaction by comparing the percent of patients who rated the overall visit as "excellent"19 between patients with and without advance directive discussions. After adjusting for the variables shown in Table 3, the visit was rated "excellent" for $44 \%$ of visits where there were discussions that day compared with $35 \%$ for visits without discussions $(P=.24)$. For visits where there were prior discussions, the adjusted percentage rated excellent was 51\% compared with $34 \%$ for visits without prior discussions $(P=.003)$.

\section{DISCUSSION}

These data suggest that elderly, chronically ill patients are more satisfied with their primary care physicians and the care they deliver when advance directives are discussed. This effect on satisfaction with the visit was greater for prior discussions than with discussions held on the day that satisfaction was assessed. This suggests that the discussion initiates thinking about advance directives by the patient that, over time, increases appreciation of the physician and the primary care he or she delivers.

A second finding was that prior discussions about advance directives improved satisfaction with the visit but not with the physician. This seems counterintuitive; greater satisfaction with the visit because of a discussion with the physician should be accompanied by greater satisfaction with the physician. In earlier analyses, we found a significant multivariable association between prior advance directive discussions and physician satisfaction; 
however, this effect became nonsignificant when we controlled for the number of prior contacts between study patients and their current primary care providers. This latter variable controls for doctor-patient familiarity, which we believe is the strongest correlate with the strength of the doctor-patient relationship. Because dissatisfied patients in the practice we studied have been shown to seek new providers, ${ }^{24}$ controlling for the length of the doctor-patient relationship may be such a strong predictor of patient satisfaction that it reduced the sensitivity of our multivariable model for any additional effects of prior discussions of advance directives.

The effect of prior discussions of advance directives on visit satisfaction was sizable: $51 \%$ of patients rated primary care visits as "excellent" when the patient and physician had discussed advance directives at a previous visit, compared with $34 \%$ of the visits where there were no prior advance directive discussions. This $17 \%$ difference is similar to the $16 \%$ difference seen in the MOS study where $65 \%$ of patients gave "excellent" ratings of their visits to physicians in fee-for-service solo practice compared with $49 \%$ of patients receiving HMO care. ${ }^{19}$ These results should encourage primary care physicians to discuss advance directives with their primary care patients, especially those who are elderly or chronically ill.

The effects of advance directive discussions on patient satisfaction remained even after controlling for strong correlations between patients' baseline satisfaction and other patient, physician, and practice factors. Caring for fewer patients on a particular day (and presumably having more time to spend with the patient) was a strong multivariable correlate of both physician and visit satisfaction. This suggests that the pressures being placed on primary care physicians to see an increasing number of patients may result in patients who are less satisfied with their care. These time pressures may also discourage primary care physicians from taking the time to discuss advance care issues with their patients. Yet it is particularly important that physicians hold such discussions in the primary care environment during routine visits, when patients are less likely to be acutely ill and can carefully explore end-of-life care issues with a physician they know and likely trust. Waiting until the patient is acutely ill and admitted to the hospital is not likely to result in productive discussions. This is evidenced by the SUPPORT study where an intensive, multifactorial intervention had no effect on the completion of advance directive forms by patients admitted to intensive care. ${ }^{25}$

Our results may have been confounded by physicians' communication skills: it is possible that physicians who are better communicators would also be more likely to both initiate advance directive discussions and have higher patient satisfaction ratings. In this study, we had no direct measure of physicians' ability to communicate and thus cannot rule out this possibility. However, it is likely that the more experienced faculty physicians would have had stronger, more polished communication skills than residents. Yet there was no significant effect of physician rank on patients' satisfaction with their physicians or visits.

This study has some limitations that need to be considered. It was performed in an academic primary care general internal medicine practice. The results may not be generalizable to other specialties or to nonacademic settings. However, it is likely that patients cared for by community-based physicians would have more stable relationships with their physicians than patients in academic settings, where residents have short tenures. There were no differences in the results for our faculty physicians, who maintain panels of patients in a private practice model, compared with residents. Therefore, there is every reason to expect that, in stable relationships with patients in the community, nonacademic physicians initiating advance directive discussions would realize the same beneficial effects on patient satisfaction as we found in our academic practice. Our results do have direct applicability to academic primary care practices which, now more than ever, are competing for their patients and so must pay greater attention to, and enhance where possible, the satisfaction of their patients. ${ }^{26}$ Academic practices can no longer rely on "captured" patients who have few alternatives for receiving primary care.

Another limitation was our inability to assess exactly what occurred within advance care discussions that might have enhanced patient satisfaction. It is possible that it was the increased attention to personal issues by the physician during such discussions that increased patient satisfaction, rather than the focus of the discussion on end-of-life care. Although we considered recording doctor-patient encounters to ascertain the cause of any effects on the doctor-patient relationship, doing so was beyond the scope of this study. In the end, it may not really matter whether it was the content of the discussions or the time spent discussing these issues that increased satisfaction with the physician and the visit, because one cannot have the former without the latter.

Discussions of advance directives are appropriate for primary care patients in general and especially for the elderly and chronically ill patients in this study, and when such discussions were held, the patients were more satisfied with their primary care and their physicians. We therefore urge primary care practices to encourage their physicians to initiate discussions about advance directives, especially with patients at risk for morbid events. These efforts seem to enhance the doctor-patient relationship, and the effects are both substantial and lasting.

The authors wish to thank the physicians and nurses of the General Medicine Practice for their unfailing support during this study. Support for this study was provided by grant R0 1-HSO7632 from the Agency for Healthcare Research and Quality (AHRQ). The opinions expressed herein do not necessarily represent those of the funding agency or the institutions employing the investigators. 


\section{REFERENCES}

1. Emanuel LL, Barry MJ, Stoeckle JD, Ettelson LM, Emanuel EJ. Advance directives for medical care-a case for greater use. N Engl J Med. 1991;324:889-95.

2. President's Commission for the Study of Ethical Problems in Medicine and Biomedical and Behavioral Research. Deciding to Forego Life-Sustaining Treatment: A Report on the Ethical, Medical, and Legal Issues in Treatment Decision. Washington, DC: US Government Printing Office; 1983. Publication 0-425748 .

3. Wenger NS, Oye RK, Bellamy PE, et al. Prior capacity of patients lacking decision making ability early in hospitalization: implications for advance directive administration. J Gen Intern Med. 1994;9:539-43.

4. Gillick M. The high costs of dying: a way out. Arch Intern Med. 1994;154:2134-37.

5. Chambers CV, Diamond JJ, Perkel RL, Lasch LA. Relationship of advance directives to hospital charges in a Medicare population. Arch Intern Med. 1994; 154:541-7.

6. Lurie N, Pheley AM, Miles SH, Bannick-Mohrland S. Attitudes toward discussing life-sustaining treatments in extended care facility patients. J Am Geriatr Soc. 1992;40:1205-8.

7. Gamble ER, McDonald PJ, Lichstein PR. Knowledge, attitudes, and behavior of elderly persons regarding living wills. Arch Intern Med. 1991;151:277-80.

8. Broadwell AW, Boisaubin EV, Dunn JK, Engelhardt HT. Advance directives on hospital admission: a survey of patient attitudes. South Med J. 1993;86:165-8.

9. Dexter PD, Wolinsky FD, Gramelspacher GP, et al. Effectiveness of computer-generated reminders for increasing discussions about advance directives and completion of advance directive forms. A randomized, controlled trial. Ann Intern Med. 1998;128: 102-10.

10. Hanson LC, Tulsky JA, Danis M. Can clinical interventions change care at the end of life? Ann Intern Med. 1997;126:381-8.

11. Schneiderman LJ, Kronick R, Kaplan RM, Anderson JP, Langer RD. Effects of offering advance directives on medical treatments and costs. Ann Intern Med. 1992;117:599-606.

12. Kellogg FR, Crain M, Corwin J, Brickner PW. Life-sustaining interventions in frail elderly persons. Talking about choices. Arch Intern Med. 1992;152:2317-20.
13. Molloy DW, Guyatt GH, Russo R, et al. Systematic implementation of an advance health care directive in nursing homes. JAMA. 2000;283:1437-44.

14. Tierney WM, Miller ME, Hui SL, McDonald CJ. Practice randomization and clinical research: the Indiana experience. Med Care. 1991;29:JS57-JS64.

15. McDonald CJ, Overhage JM, Tierney WM, et al. The Regenstrief Medical Record System: a quarter century experience. Int J Med Informat. 1999;54:225-53.

16. McDonald CJ. Action-Oriented Decisions in Ambulatory Medicine. Chicago: Yearbook Medical Publishers; 1981.

17. Pfeiffer E. A short portable status questionnaire for the assessment of organic brain deficit in elderly patients. J Am Geriat Soc. 1975;10:433-41.

18. Webster GD. Final Report of the ABIM Patient Satisfaction Project. Philadelphia: American Board of Internal Medicine; 1988.

19. Rubin HR, Gandek B, Rogers WH, Kosinski M, McHorney CA, Ware JE Jr. Patients' ratings of outpatient visits in different practice settings: results from the Medical Outcomes Study. JAMA. 1993;270:835-40.

20. Stump TE, Dexter PR, Tierney WM, Wolinsky FD. Measuring patient satisfaction with physicians among older and diseased adults in the outpatient setting: a comparison of three instruments. Med Care. 1995;33:958-72.

21. Ware JE Jr, Kosinski M, Keller SD. SF-36 Physical and Mental Health Summary Scales: A User's Manual. Boston: The Health Institute; 1994.

22. Danis M, Southerland LI, Garrett JM, et al. A prospective study of advance directives for life-sustaining care. N Engl J Med. 1991;324:882-8.

23. Liang KY, Zeger SL. Longitudinal data analysis using generalized linear models. Biometrika. 1986;73:13-22.

24. Harris LE, Swindle RW, Mungai SM, Weinberger M, Tierney WM. Measuring patient satisfaction for quality improvement. Med Care. 1999;37:1207-25.

25. The SUPPORT Principal Investigators. A controlled trial to improve care for seriously ill hospitalized patients: the study to understand prognoses and preferences for outcomes and risks of treatments (SUPPORT). JAMA. 1995;274:1591-8.

26. Fein R. The academic health center: some policy reflections. JAMA. 2000;283:2436-7. 


\section{APPENDIX}

\section{Physician Satisfaction Instrument from the American Board of Internal Medicine ${ }^{14}$}

In terms of your satisfaction, how would you rate the doctor that you saw first today on each of the following?

1. How do you rate Dr. on telling you everything; being truthful [up-front, frank; not keeping things from you that you should know] *

2. Greeting you warmly; being friendly [calling you by the name you prefer; never rude or crabby]

3. Treating you like you are on the same level; never talking down to you [like a child]

4. Letting you tell your story; listening carefully; asking thoughtful questions; not interrupting while you're talking

5. How do you rate Dr. ___ on showing interest in you as a person [not acting bored or ignoring what you have to say]

6. Telling you during the physical about what $\mathrm{s} /$ he is going to do and why; telling you what $\mathrm{s} /$ he finds?

7. Discussing choices with you; asking your opinion [offering choices and letting you help decide what to do; asking what you think before telling you what to do]

8. Encouraging you to ask questions, and answering them clearly; never avoiding your questions or lecturing to you

9. Explaining what you need to know about your problems, how and why they occurred, and what to expect next

10. Using words you can understand when explaining your problems and treatment [explaining any technical medical terms in plain language]

\begin{tabular}{lllll} 
Excellent & Very Good & Good & Fair & Poor \\
$\square$ & $\square$ & $\square$ & $\square$ & $\square$ \\
Excellent & Very Good & Good & Fair & Poor \\
$\square$ & $\square$ & $\square$ & $\square$ & $\square$ \\
Excellent & Very Good & Good & Fair & Poor \\
$\square$ & $\square$ & $\square$ & $\square$ & $\square$ \\
Excellent & Very Good & Good & Fair & Poor \\
$\square$ & $\square$ & $\square$ & $\square$ & $\square$ \\
Excellent & Very Good & Good & Fair & Poor \\
$\square$ & $\square$ & $\square$ & $\square$ & $\square$ \\
Excellent & Very Good & Good & Fair & Poor \\
$\square$ & $\square$ & $\square$ & $\square$ & $\square$ \\
Excellent & Very Good & Good & Fair & Poor \\
$\square$ & $\square$ & $\square$ & $\square$ & $\square$ \\
Excellent & Very Good & Good & Fair & Poor \\
$\square$ & $\square$ & $\square$ & $\square$ & $\square$ \\
Excellent & Very Good & Good & Fair & Poor \\
$\square$ & $\square$ & $\square$ & $\square$ & $\square$ \\
Excellent & Very Good & Good & Fair & Poor \\
$\square$ & $\square$ & $\square$ & $\square$ & $\square$ \\
& & & & \\
\hline
\end{tabular}

* Interviewer only reads what is in the square brackets when the patient asks for clarification.

\section{Visit Satisfaction Instrument from the Medical Outcomes Study ${ }^{15}$}

Here are some questions about the visit you just made and the doctor you saw first today. In terms of your satisfaction, how would you rate each of the following?

1. The time spent with the doctor

2. The doctor's explanation of what was done for you

3. The doctor's skills (thoroughness, carefulness, competence)

4. The doctor's personal manner (courtesy, respect, sensitivity, friendliness)

5. This visit overall

\begin{tabular}{lllll} 
Excellent & Very Good & Good & Fair & Poor \\
$\square$ & $\square$ & $\square$ & $\square$ & $\square$ \\
Excellent & Very Good & Good & Fair & Poor \\
$\square$ & $\square$ & $\square$ & $\square$ & $\square$ \\
Excellent & Very Good & Good & Fair & Poor \\
$\square$ & $\square$ & $\square$ & $\square$ & $\square$ \\
Excellent & Very Good & Good & Fair & Poor \\
$\square$ & $\square$ & $\square$ & $\square$ & $\square$ \\
Excellent & Very Good & Good & Fair & Poor \\
$\square$ & $\square$ & $\square$ & $\square$ & $\square$ \\
\hline
\end{tabular}

